\title{
Alterstice
}

Revue internationale de la recherche interculturelle

International Journal of Intercultural Research

Revista International de la Investigacion Intercultural

\section{Donner la parole}

\section{Yvan Leanza}

Volume 7, numéro 2, 2017

URI : https://id.erudit.org/iderudit/1052563ar

DOI : https://doi.org/10.7202/1052563ar

Aller au sommaire du numéro

Éditeur(s)

Alterstice

ISSN

1923-919X (numérique)

Découvrir la revue

Citer ce document

Leanza, Y. (2017). Donner la parole. Alterstice, 7(2), 1-2.

https://doi.org/10.7202/1052563ar d'utilisation que vous pouvez consulter en ligne.

https://apropos.erudit.org/fr/usagers/politique-dutilisation/ 
ÉDITORIAL

\section{Donner la parole}

Yvan Leanza ${ }^{1}$

Je constate, en tant que directeur d'Alterstice, que la majorité des articles soumis à la revue sont des comptes rendus de recherches qualitatives. C'est une grande richesse dans la mesure où les chercheurs ont justement fait d'incommensurables efforts pour aller chercher la parole (ou observer) des personnes de groupes souvent peu entendus dans l'environnement médiatique quotidien. Ce travail, donner la parole à ceux qui ne l'ont pas, est l'un des principes du métier de chercheur selon l'anthropologue Bruno Latour (2001). Comme le biologiste spécialiste de la canopée peut donner la parole aux arbres, aux oiseaux et aux insectes de la cîme des arbres, ou le physicien fait parler les particules élémentaires qui constituent la matière ou les astres de l'univers, l'interculturaliste donne la parole aux migrants, réfugiés, professionnels qui travaillent auprès d'eux, employeurs qui les recrutent, politiques qui établissent les lois... L'interculturaliste écoute les personnes qui vivent des formes d'interculturalité au quotidien. Par leurs analyses de cette parole, les chercheurs permettent de contrer les préjugés et stéréotypes habituellement véhiculés sur les enjeux interculturels et, peut-être, transformer certains aspects de la société. C'est mon espoir.

Alors, y a-t-il problème ? Malheureusement, j'en vois un dans la façon de présenter la parole des participants aux recherches. C'est un problème qui se corrige facilement, mais je suis souvent surpris de l'apparent manque de considération des auteurs quant à la présentation de cette précieuse parole qui leur permet de construire des théories et faire avancer leurs idées. Ce qui manque presque systématiquement, et que je vois comme une forme de déshumanisation des participants dans le processus de la recherche, c'est le rattachement de la parole à la personne qui l'a prononcée. La majorité des articles soumis présentent les dires des participants sans expliciter qui parle!

Je comprends les enjeux éthiques rattachés à ce point: nous avons obligation de protéger les personnes qui participent à nos recherches, à moins, dans quelques cas particuliers, qu'elles aient choisi de ne pas rester anonymes. Cette obligation implique de devoir faire des choix dans la présentation des citations. Les différentes normes pour l'écriture scientifique, par exemple celles de l'American Psychological Association APA qu'Alterstice applique pour les notices bibliographiques, proposent des lignes directrices pour orienter ces choix (voir par exemple le billet de blogue de Lee et Hume-Pratuch, 2013, sur le sujet). Sans être trop spécifique, il est possible de mettre des informations très générales, par exemple une tranche d'âges plutôt que l'âge précis, ou une région du monde plutôt qu'une nationalité. Et cela peut se faire par un code déterminé au départ pour alléger le texte. S'il demeure vraiment impossible de donner ce type d'information sans remettre en cause l'anonymat, cela peut être expliqué en détail dans l'article. Bien que cette approche normative pour protéger l'anonymat soit nécessaire, ce n'est pas la seule qui est mentionnée par les chercheurs lorsqu'ils sont interrogés sur ce sujet. En effet, Corden et Sainsbury (2006) montrent que les choix des auteurs pour indiquer au lecteur qui s'exprime sont dépendants de plusieurs facteurs : le domaine, l'objectif de la recherche, l'utilité de la citation et la lisibilité du texte. II me semble que l'on peut aller un peu plus loin dans la réflexion.

Dans toute recherche en science sociales, une présentation des participants est de mise. Cette présentation, même succincte, donne quelques informations sur les participants : âge, genre, langues parlées, nationalités... Or, dans mon expérience de directeur de la revue, une fois cette présentation générale effectuée, les auteurs " oublient » 
de préciser à qui revient la parole citée. Il me semble que cet oubli va à l'encontre du principe même de donner la parole. Ce faisant, ce sont les particularités de la personne qui disparaissent, qui sont noyées dans l'ensemble " participant ». Parfois, dans le cas de petits groupes facilement reconnaissables, c'est compréhensible. II s'agit encore une fois de maintenir l'anonymat et la confidentialité. Cependant, dans la majorité des cas, indiquer sommairement si la personne dont on rapporte les paroles est une femme ou un homme, âgée de ..., parlant ..., ou tout autre information en lien avec le phénomène à l'étude n'est pas un luxe. Cet effort permet de réincarner la parole : ce ne sont plus des sujets anonymes, mais bien des humains qui ont confié une part de leur expérience vécue qui parlent. C'est une forme de reddition respectueuse : le chercheur ne s'approprie pas cette parole, il la rend à la personne qui l'a prononcée. C'est aussi une façon de donner au lecteur une représentation de la personne qui parle.

Le présent numéro a été concocté par une équipe intercontinentale d'éditrices et éditeur invités : Kalidou Sy de l'Université Gaston Berger de Saint-Louis au Sénégal et Pia Stalder, Josianne Veillette et Aline Gohard-Radenkovic des universités de la Suisse italienne et de Fribourg (Suisse). Ils ont su réunir un beau mélange de textes qui traite de la diversité sous des formes variées sur les deux continents, et qui ont en commun d'analyser les réponses des institutions et des intervenants à cette diversité.

Bonne lecture!

\section{Références}

Corden, A. et Sainsbury, R. (2006). Using verbatim quotations in reporting qualitative social research: researchers' views. University of York (GB) : Social Policy Research Unit. https://www.york.ac.uk/inst/spru/pubs/pdf/verbquotresearch.pdf

Latour, B. (2001). Le métier de chercheur. Regard d'un anthropologue ( $2^{\mathrm{e}}$ éd.). Paris : Éditions Quae.

Lee, C. et Hume-Pratuch, J. (2013). Let's talk about research participants [Billet de blogue]. http://blog.apastyle.org/apastyle/2013/08/lets-talk-about-research-participants.html

\section{Rattachement de l'auteur}

${ }^{1}$ Université Laval, Québec, Canada

\section{Correspondance}

alterstice@gmail.com

Pour citer cet article

Leanza, Y. (2017). Donner la parole [Éditorial]. Alterstice, 7(2), 1-2. 